\title{
RELAÇÕES ENTRE COMPREENSÃO LEITORA, ATITUDES DE LEITURA E ESTRATÉGIAS DE APRENDIZAGEM EM UNIVERSITÁRIOS
}

Nilza de Matos Presto - Universidade Salgado de Oliveira/Juiz de Fora Karen Cristina Alves Lamas - Universidade Salgado de Oliveira/Juiz de Fora

Nilza de Matos Presto

Psicóloga e pós-graduanda em Terapia Cognitivo-Comportamental pela Universidade Salgado de Oliveira -

UNIVERSO/Juiz de Fora

Karen Cristina Alves Lamas

Psicóloga pela Universidade Federal de Juiz de Fora/UFJF, doutora em psicologia pela Universidade São Francisco, docente do curso de graduação em psicologia da Universidade Salgado de Oliveira - UNIVERSO/Juiz de Fora

\section{Correspondência:}

Karen Cristina Alves Lamas

Av. dos Andradas, 731, Morro da Glória, 36036-000, Juiz de Fora - MG.

Telefone: (32) 3311-9917

E-mail: karen_lammas@yahoo.com.br

E-mail: nilza.psi28@gmail.com 


\section{Resumo}

O objetivo deste estudo foi investigar o nível de compreensão leitora, atitudes de leitura e uso de estratégias de aprendizagem de universitários, e identificar correlações entre essas variáveis comparando os escores obtidos entre grupos de ingressantes e concluintes. Participaram 107 estudantes de psicologia de uma universidade privada do interior de Minas Gerais. Para coleta dos dados foram utilizados: texto preparado segundo a Técnica de Cloze, Escala de Atitudes de leitura e Escala de Estratégias de Aprendizagem para Estudantes Universitários (EEA - U). Os resultados demonstraram nível de compreensão textual aquém do desejado, correlação entre o uso de estratégias de aprendizagem e atitudes em leitura e ausência de diferenças significativas entre os grupos. Verificou-se a necessidade de programas de diagnóstico e intervenção para promoção da leitura proficiente, necessária à qualidade da formação profissional.

Palavras-chave: habilidades de leitura; aprendizagem; teste de cloze; estudantes universitários

\section{Abstract}

The aim of this study was to investigate the level of reading comprehension, reading attitudes and use of learning strategies in college students, and to identify correlations between these variables by comparing the scores obtained between groups of freshmen and seniors. 107 psychology students from a private university in the interior of Minas Gerais participated. For data collection were used: text prepared according to the Cloze Technique, Reading Attitudes Scale and Learning Strategies Scale for University Students (EEA - U). The results showed a lower level of textual comprehension than desired, a correlation between the use of learning strategies and reading attitudes and the absence of significant differences between the groups. The need for diagnostic and intervention programs to promote proficient reading, necessary for the quality of vocational training, was verified.

Keywords: reading skills; learning; cloze testing; college students 


\section{Introdução}

A leitura é uma habilidade linguística muito presente na vida do ser humano que proporciona uma melhor inserção na sociedade, possibilitando a aquisição do saber e sua utilização de forma adequada e produtiva. A capacidade de ler é um fenômeno complexo que envolve diversos processos mentais, como: linguagem, percepção, pensamento, memória, aprendizagem, motivação, inteligência, dentre outros. Por meio da leitura é possível desenvolver a competência da reflexão, ampliar a percepção em relação a óticas distintas, aumentar o vocabulário, estimular a criatividade e melhorar a comunicação. No entanto, estudantes de diferentes níveis de escolaridade têm enfrentado dificuldades na compreensão de textos (Alcará \& Santos, 2013; Cantalice \& Oliveira, 2009; Calderon-Ibanez \& Quijano-Peñuela, 2010; Lawi, 2015; Sternberg, 2000).

No âmbito do ensino superior, a leitura representa uma ferramenta essencial para a formação teórica e prática dos universitários, pois é o principal meio de acesso à informação e ao conhecimento inerente à futura profissão. Desse modo, na última etapa de escolarização formal, espera-se do aluno um nível independente de leitura, com capacidade de compreender e analisar as informações lidas, tornando-se um sujeito ativo, crítico e envolvido com as questões sociais e políticas do meio em que está inserido. O desenvolvimento da competência em leitura é importante para qualidade da formação no ensino superior, visto que esse nível de ensino permite aos seus egressos a imediata inserção no mercado de trabalho (Cantalice \& Oliveira, 2009; Lawi, 2015; Santos, 1997; Santos, Vendramini, Suehiro, \& Santos, 2006).

De acordo com Alcará e Santos (2013), a capacidade de compreensão de leitura e o uso adequado de estratégias de aprendizagem são fatores que influenciam o desempenho acadêmico na universidade. Neste sentido, Oliveira, R. e Oliveira, K. (2007) afirmam que para que a 
aprendizagem ocorra são necessárias condições adequadas de estudo e compreensão dos textos lidos. Entre as estratégias de estudo, neste caso, destacam-se aquelas direcionadas à leitura. Cantalice e Oliveira (2009) observaram uma correlação positiva estatisticamente significativa entre o uso de estratégias de leitura e compreensão textual, num estudo com universitários.

Estratégias de leitura são os diferentes métodos que o estudante utiliza para ler e compreender a informação escrita, portanto são flexíveis e adaptadas às características de cada texto, de forma a garantir o alcance dos objetivos definidos (Cantalice \& Oliveira, 2009). Para um bom desempenho acadêmico, Gonçalves (2008) considera importante a utilização de estratégias de leitura que favoreçam a compreensão de textos, citando como exemplos: a identificação das ideias principais do texto, tanto em função dos objetivos do autor, como dos próprios objetivos da leitura; a sumariação, que significa a construção de um novo texto coeso e coerente a partir de uma síntese do texto lido; realização de inferências; e elaboração de questões sobre o texto. O autor destaca ainda, estratégias metacognitivas, as quais se relacionam à capacidade de o estudante estar consciente do seu próprio processo de aprendizagem, através de uma autoavaliação em que é capaz de monitorar o seu processo de compreensão modificando as estratégias utilizadas quando as percebe ineficazes.

Oliveira, R. e Oliveira, K. (2007) identificaram num estudo com universitários ingressantes que as três estratégias de estudo mais utilizadas são: leitura em voz alta, resumo com as próprias palavras e resumo com as palavras do texto, o que demonstra que esses estudantes não aprenderam a aprender e se utilizam de estratégias simples, sem planejar e monitorar a própria aprendizagem. Além disso, os alunos afirmaram não possuírem o hábito de lerem livros não obrigatórios para aprendizagem acadêmica, compreendendo o processo de ler como obrigação para sua própria 
formação, uma concepção inadequada da leitura, para alunos universitários, a qual influencia na qualidade da formação de profissionais críticos e competentes.

Apesar da importância da competência em leitura para os universitários, diversos estudos (Alcará \& Santos, 2013, 2015; Oliveira, Cunha, \& Santos, 2015; Cantalice \& Oliveira, 2009; Figueiredo, Minervino, Pereira, \& Silveira, 2016; Oliveira, 2011; Santos, et al., 2006) apontam que os estudantes do ensino superior possuem um repertório ineficaz de estratégias de estudo e um nível de leitura muito aquém do desejado. O que interfere negativamente no desempenho acadêmico, prejudicando a aquisição do conhecimento, a qualidade da formação, e consequentemente a atuação do futuro profissional na sociedade.

Analisando a compreensão de leitura de universitários ingressantes e concluintes, Figueiredo et al. (2016) e Santos et al. (2006) não encontraram diferenças significativas, o que significa, que as dificuldades com as quais os estudantes iniciam os cursos permanecem durante toda a formação. O estudo de Figueiredo et al. (2016) apontou também, inexistência de diferenças significativas entre ingressantes e concluintes quanto ao uso de estratégias de aprendizagem. Tais dados são preocupantes porque demonstram limitações no ensino superior e despreparo técnico para o exercício futuro da profissão (Cantalice \& Oliveira, 2009).

O baixo desempenho em leitura dos estudantes universitários deve ser considerado em sua complexidade, como consequência de diversos fatores. Ressalta-se, por exemplo, que os alunos ingressam na universidade trazendo consigo muitas dificuldades devido a deficiências na educação básica, a qual não está formando leitores competentes e autônomos; desconhecimento ou uso inadequado de estratégias de estudo; falta de autocontrole sobre as questões relacionadas à aprendizagem, vocabulário e conhecimento prévios limitados; baixa autoeficácia; dificuldades de concentração; hábitos inadequados de estudo, e escassez de tempo para dedicação aos estudos 
(Alcará \& Santos, 2013, 2015; Calderon-Ibanez \& Quijano-Peñuela, 2010; Santos, 1997; Gonçalves, 2008; Oliveira, 2011; Silva \& Witter, 2008).

Muitos são os fatores que interferem na aprendizagem e no desempenho acadêmico, como por exemplo, o estilo cognitivo, a regulação emocional, a motivação, as estratégias e as condições de estudo (Alcará, 2012; Bortelo \& Boruchovitch, 2013; Tinajero, Lemos, Araújo, Ferraces, \& Páramo, 2012). Além desses fatores, os hábitos de leitura e as dificuldades que os estudantes encontram no processo de compreensão dos textos podem ser pensados em relação às atitudes que os mesmos apresentam diante do ato de ler (Oliveira, Santos, \& Oliveira, 2007).

Para Oliveira et al. (2007) a compreensão de um texto depende de uma disposição para lêlo. De acordo com Rodrigues (1973) conhecer as atitudes de uma pessoa em relação a determinado objeto, pode contribuir para prever seus comportamentos diante dos mesmos. $\mathrm{O}$ autor define a atitude como um conjunto de crenças e cognições, dotado de carga afetiva pró ou contra um objeto definido, que predispõe a uma ação coerente com as cognições e afetos acerca do objeto. Assim as atitudes seriam formadas por três componentes: cognitivo, afetivo e comportamental. Possuem ainda capacidade de influenciar fenômenos psicológicos como: motivação, percepção e aprendizagem.

Para Santos (2004) as dificuldades de compreensão em leitura podem gerar um círculo vicioso em que o estudante passa a ter uma atitude de evitação diante da leitura perdendo assim a oportunidade de desenvolver as habilidades a partir da prática, o que mantém as limitações que fazem diminuir a motivação para atividade de leitura se transformando num obstáculo para aprendizagem efetiva. Em um estudo com universitários, Oliveira (2008) buscou compreender as relações entre compreensão de leitura, atitudes de leitura e desesperança, com a hipótese de que os alunos com sentimentos de desesperança tenham também atitudes negativas diante da leitura e 
desempenho insatisfatório na compreensão de leitura. Os resultados da pesquisa demonstraram relação significativa e negativa entre desesperança e atitudes, ou seja, quanto mais atitudes positivas diante da leitura, menor a pontuação na escala de desesperança.

Considerando a relevância da leitura no ensino superior, estudos que investiguem a compreensão textual de universitários e o uso eficaz de estratégias de estudo podem contribuir para que a universidade possa desenvolva estratégias de diagnóstico e intervenção, como programas remediativos para auxiliar os estudantes a superarem as dificuldades, melhorando a qualidade do processo ensino-aprendizagem (Alcará \& Santos, 2013, 2015; Santos et al., 2006; Oliveira, 2011). Dessa forma, o presente estudo tem por objetivo investigar a relação entre o nível de compreensão leitora, atitudes de leitura e uso de estratégias de aprendizagem de universitários. Mais especificamente pretende-se (a) verificar o nível de compreensão leitora dos estudantes universitários do curso de psicologia de uma da Universidade particular do interior de Minas Gerais, (b) identificar as estratégias de aprendizagem empregadas pelos estudantes, (c) conhecer as atitudes dos estudantes diante da leitura, (d) comparar o nível de compreensão leitora, as atitudes de leitura e o uso de estratégias de estudo dos estudantes ingressantes e concluintes, e por fim, (e) investigar a relação entre compreensão leitora, atitudes de leitura e uso de estratégias de aprendizagem.

Fundamentando-se em outros estudos, a hipótese inicial é de que os universitários apresentem um nível de compreensão leitora inferior ao desejado (Alcará \& Santos, 2013, 2015; Figueiredo et al., 2016, Oliveira, 2008; Oliveira, 2011; Oliveira et al., 2015; Santos, Suehiro, \& Oliveira, 2004; Santos, et al., 2006). Espera-se encontrar uma correlação positiva entre o nível de compreensão leitora e as atitudes de leitura, uma vez que atitudes positivas podem facilitar a compreensão da informação lida (Oliveira, 2008). Desse modo aventa-se a hipótese de que atitudes 
positivas contribuam para aumentar o hábito de leitura que por sua vez afetaria o desempenho na compreensão, enquanto que o estudante com atitudes negativas diante da leitura seria mais propicio a evitá-la preservando suas dificuldades. Também se espera que o nível de compreensão leitora apresente correlação positiva com as estratégias de aprendizagem (Cantalice \& Oliveira, 2009).

Também se espera que o nível de compreensão leitora apresente correlação positiva com as estratégias de aprendizagem (Cantalice \& Oliveira, 2009), denotando que o aluno que utiliza mais estratégias de aprendizagem, tem mais autonomia nos estudos e uma melhor compreensão textual, assim como melhores atitudes em relação à atividade de ler. Quanto à relação entre estratégias de aprendizagem e as atitudes de leitura, a expectativa é de que haja uma correlação positiva. Considera-se tal investigação importante, visto que não foram encontradas pesquisas que focalizassem a relação entre essas variáveis. Em relação à comparação por grupos, a expectativa é que não haja diferença significativa entre os grupos quanto à compreensão leitora, nem quanto ao uso de estratégias, conforme revisão de literatura (Figueiredo, et al., 2016; Santos, et al., 2006).

\section{Método}

\section{Participantes}

A seleção da amostra foi estabelecida por conveniência, sendo a escolha do curso de psicologia, pensada em termos da exigência de leitura feita aos estudantes. Participaram desta pesquisa 107 estudantes universitários, ingressantes $(n=69)$ e concluintes $(n=38)$ do curso de psicologia, período noturno de uma Universidade particular do interior de Minas Gerais. O sexo feminino representou $83 \%(\mathrm{n}=89)$ da amostra e o sexo masculino $16 \%(\mathrm{n}=18)$. A média de idade foi de 25,63 anos ( $\mathrm{DP}=8,123)$. A idade mínima foi de 18 anos e a máxima de 53 anos, sendo que três participantes não informaram a idade. 


\section{Instrumentos}

Para o alcance dos objetivos propostos neste estudo foram utilizados três instrumentos para coleta de dados: o Teste de Cloze, uma Escala de Atitudes em leitura e a Escala de Estratégias de Aprendizagem para estudantes universitários (EEA - U).

O Teste de Cloze é um instrumento para avaliar a compreensão de leitura. A Técnica, elaborada por Taylor em 1953, consiste na omissão de todos os quintos vocábulos do texto substituindo-os por traços de tamanho proporcional a palavra omitida. Neste estudo utilizou-se o texto Desentendimento (1995) de Luís Fernando Veríssimo, preparado segundo a técnica de Cloze, o qual tem sido usado em outras pesquisas com universitários, apresentando evidências de validade e precisão para avaliação de compreensão em leitura, com bom nível de consistência interna $(K R$ $20=0,84)($ Oliveira \& Santos, 2005; Santos, et al., 2002).

O texto contém cerca de 250 vocábulos e 46 omissões. Utilizou-se o critério de correção literal, no qual são considerados acertos o preenchimento com a palavra idêntica a omitida. A pontuação pode variar de 0 a 46, sendo atribuído valor 1 para os acertos e 0 para os erros. Para interpretar os resultados do Teste de Cloze, Bornuth (1968 apud, Oliveira \& Santos, 2008) estabelece 3 níveis de desempenho, o primeiro é o nível de frustração, no qual o estudante atinge até $44 \%$ de acertos, indica que não houve compreensão do texto, o segundo é o nível instrucional, a pontuação vai de 44,1 a 57\% de acertos, indica abstração apenas suficiente para compreensão e o terceiro é o nível independente, com pontuação superior a 57\%, que indica compreensão crítica, criativa e autônoma do texto.

A Escala de Atitudes em leitura baseada no questionário elaborado por Silva e Naher (1981) adaptada e utilizada por Oliveira (2008) e Oliveira et al. (2007) em estudos com universitários. Possui evidências de validade fatorial e índices aceitáveis de consistência interna, 
alpha de Cronbach igual a 0,89, identificadas no estudo de Oliveira et al. (2007). A escala contém 30 afirmativas e os estudantes devem assinalar para cada item uma opção entre: Concordo plenamente (CP), Concordo (C), Discordo (D) e Discordo plenamente (DP). Sendo atribuídos os seguintes valores respectivamente, 4 (CP), 3 (C), 2 (D) e 1 (DP). O escore total pode chegar a 120 representando a soma dos valores de cada item.

A Escala de Estratégias de Aprendizagem para estudantes universitários (EEA - U) de Santos e Boruchovitch (2015) em sua terceira versão, que apresenta consistência interna com o alfa de Cronbach de 0,87. É composta de 35 itens, agrupados em três fatores, sendo que fator 1 avalia estratégias cognitivas e metacognitivas, o fator 2 - avalia estratégias de autorregulação dos recursos internos e contextuais e o fator 3 - avalia estratégias de autorregulação social. Os itens estão organizados em forma de escala Likert, com 4 opções de respostas: sempre, às vezes, raramente e nunca, atribuindo os seguintes valores respectivamente: 3, 2, 1 e 0 pontos. Assim, a pontuação da escala pode variar de 0 a 105 pontos. Novas evidencias de validade para essa escala foram identificadas por Mohallem (2016) ao encontrar existência de correlação com um instrumento que avalia a autoeficácia acadêmica, um construto relacionado.

\section{Procedimentos e Cuidados éticos}

Os cuidados éticos da presente pesquisa estão em conformidade com as recomendações da resolução 466/12 do Conselho Nacional de Saúde. A coleta de dados se iniciou após aprovação do projeto pelo Comitê de Ética em pesquisa, da Universidade Salgado de Oliveira. Foi estabelecido um bom rapport antes da aplicação dos instrumentos, com esclarecimento dos objetivos da pesquisa, da garantia do anonimato e da possibilidade de desistência a qualquer momento da pesquisa. Os instrumentos foram preenchidos pelos participantes que assinaram o Termo de Consentimento Livre e Esclarecido (TCLE). 
A aplicação dos instrumentos ocorreu de forma coletiva em sala de aula, com prévia autorização da instituição e dos docentes e em horários agendados. Quanto à ordem, primeiramente foi aplicado o Teste de Cloze, em seguida a Escala de Atitudes em Leitura e finalmente a Escala de Estratégias de Aprendizagem. A aplicação ocorreu no final do mês de agosto e início do mês de setembro de 2018. Cada aplicação teve duração média de 40 minutos.

\section{Resultados/Discussão}

Os dados foram organizados em planilha e analisados por meio de estatística descritiva e inferencial. Foi utilizada a Correlação de Pearson para analisar as relações entre compreensão em leitura, atitudes de leitura e uso de estratégias de aprendizagem e o Teste $t$ de Student para analisar possíveis diferenças entre essas variáveis por grupos (ingressantes e concluintes). O nível de significância adotado foi o de 0,05 .

Conforme Tabela 1, a média de pontos da compreensão em leitura obtida pelos universitários foi baixa, sendo a pontuação mínima de 3 e a máxima de 30 pontos. Nenhum estudante conseguiu atingir a pontuação máxima de 46 pontos e a média de acertos foi inferior a 50\%. De acordo com a classificação estabelecida por Bormuth (1968, Oliveira \& Santos, 2008) a média de pontos obtida pela presente amostra se refere ao nível de frustração indicando que não houve compreensão do texto. Do total da amostra $(\mathrm{N}=107), 49$ estudantes $(45,8 \%)$ ficaram no nível de Frustração, o qual significa que não conseguem compreender o texto sem ajuda externa, 49 (45,8\%) ficaram no nível instrucional com abstração apenas suficiente para compreensão, e apenas 9 estudantes $(8,41 \%)$ ficaram no nível Independente de leitura, ou seja, que conseguem compreender o texto de forma autônoma, crítica e criativa, nível esperado para estudantes do ensino superior. Enfatiza-se que esse resultado é congruente com diversos estudos (Alcará \& Santos; 2013, 2015; Oliveira et al., 2015; Figueiredo et al., 2016, Oliveira, 2008; Oliveira, 2011; 
Santos et al., 2004; Santos et al., 2006) que demonstraram que o nível de compreensão leitora dos universitários é inferior ao desejado para compreensão adequada das informações lidas. Nessas pesquisas os estudantes obtiveram média no Cloze inferior a 50\% de acertos, sendo classificados no nível instrucional ou de frustração e também não houve nenhum participante que conseguiu atingir o total de 46 acertos

Tabela 1

Estatísticas descritivas

\begin{tabular}{lllllc}
\hline Medidas & N & Mínimo & Máximo & Média & DP \\
\hline Close & 107 & 3 & 30 & 20,45 & 5,15 \\
Autorregulação Cognitiva e & 107 & 19,00 & 67,00 & 49,44 & 9,31 \\
Metacognitiva & & & & & \\
Autorregulação dos recursos internos e & 107 & 5,00 & 23,00 & 14,85 & 3,93 \\
contextuais & & & & & \\
Autorregulação social & 107 & 1,00 & 12,00 & 7,72 & 2,61 \\
Total - Estratégias de aprendizagem & 107 & 29,00 & 100,00 & 72,01 & 12,59 \\
Sentimentos afetivos frente à leitura & 107 & 15,00 & 56,00 & 40,43 & 8,06 \\
Leitura e aprendizagem & 107 & 21,00 & 32,00 & 29,96 & 2,61 \\
Leitura escolar & 107 & 9,00 & 32,00 & 21,63 & 3,81 \\
Total - Atitudes em leitura & 107 & 46,00 & 119,00 & 92,02 & 12,66
\end{tabular}

No que tange às estratégias de aprendizagem utilizadas pelos universitários, observa-se na Tabela 1 que o resultado foi superior à média. As estratégias mais utilizadas são as de autorregulação cognitiva e metacognitva. Para Souza, (2010) as estratégias cognitivas estão relacionadas à execução de tarefas para melhor compreender e armazenar informação, como a repetição oral ou por escrito, a realização de resumos e elaboração de perguntas e respostas, a 
construção de diagramas, mapas mentais e a transformação do texto em tópicos. Já as estratégias metacognitvas são mais amplas e envolvem organização, regulação monitoramento, avaliando quando as estratégias selecionadas estão promovendo resultados efetivos ou se devem ser alteradas. As estratégias menos utilizadas pelos universitários foram as de autorregulação social, que envolvem o pedido de ajuda ao outro, como tirar dúvidas, estudar em grupo, discutir o conteúdo ou pedir um colega para tomar a matéria.

Esses resultados são coerentes com diversas pesquisas sobre uso de estratégias de aprendizagem. Alcará e Santos (2013) e Mohallem (2016) também identificaram que as estratégias mais utilizadas pelos universitários são as de autorregulação cognitiva e metacognitiva. Já as estratégias de autorregulação social foram as que apareceram com menor frequência também nas pesquisas de Silva (2012), Mohallem (2016) e Alcará e Santos (2013). Infere-se que a maior utilização de estratégias cognitivas e metacognitivas está relacionada à familiaridade que os estudantes possuem com essas estratégias enquanto a menor utilização de estratégias de autorregulação social pode ser entendida pelas características dos estudantes noturno, que em geral trabalham durante o dia todo e possuem pouco tempo para interação com os colegas (Alcará \& Santos, 2013).

Em relação às atitudes de leitura, como pode ser visto na Tabela 1, os estudantes obtiveram um resultado superior à média, sendo que a pontuação máxima foi de 119, quase a totalidade da escala. Esses dados são coerentes com os estudos de Oliveira (2008) e de Oliveira et al. (2007) nos quais os universitários apresentaram atitudes mais positivas do que negativas em relação à leitura. Porém, Oliveira (2008) destaca que a presença de atitudes positivas significa que o estudante compreende a importância do ato de ler e não que tenha competência ou hábitos adequados de leitura. Oliveira et al. (2007) também chamam a atenção para o fato de que a escala 
de atitudes não discrimina leitura digital da leitura impressa, o que pode ter levado o estudante com atitude negativa de leitura responder que a mesma é uma diversão pensando na leitura realizada pela internet. Apesar disso, Oliveira (2008) ressalta a importância de os alunos apresentarem atitudes positivas já que isso aumentaria a possibilidade de adesão aos programas propostos para aprimorar a compreensão leitora na universidade. Na sequência serão apresentadas e discutidas as diferenças de médias entre os grupos de ingressantes e concluintes, conforme ilustrado na Tabela 2.

Tabela 2

Distribuição das médias, desvios-padrão e teste $t$

\begin{tabular}{lclll}
\hline Medidas & Ingressantes & Concluintes & $t$ & $p$ \\
& Média (DP) & Média/ DP & & \\
& & & & \\
\hline Cloze & $20,19(4,63)$ & $20,92(6,03)$ & $-0,702$ & 0,484 \\
Atitudes de leitura & $90,94(13,97)$ & $93,97(9,70)$ & 1,188 & 0,238 \\
Estratégias de aprendizagem & $74,10(11,96)$ & $68,21(12,97)$ & 2,366 & 0,20
\end{tabular}

Comparando o desempenho no teste Cloze entre os estudantes ingressantes $(M=20,92)$ e concluintes $(M=20,19)$ não foi identificada diferença significativa, sugerindo que nesta amostra as dificuldades de compreensão leitora permaneceram durante os anos de formação no ensino superior. Dado preocupante que também foi constatado na pesquisa de Figueiredo et al. (2016) e Santos et al. (2006). Os estudantes alcançaram um resultado muito aquém do desejado, o que denota dificuldades para compreensão do texto lido, influenciando negativamente o desempenho acadêmico e a qualidade da formação do futuro profissional. Tais achados estão em consonância com as pesquisas de Oliveira e Santos (2008), Alcará e Santos (2013), Figueiredo et al. (2016), Oliveira (2011) e Santos et al. (2006). 
Em relação às atitudes em leitura, o resultado dos grupos foi superior à média, com pontuação um pouco melhor para os alunos concluintes, contudo a diferença não foi estatisticamente significativa. Esse resultado indica que os alunos têm uma atitude positiva em relação à leitura, compreendendo o valor da mesma para formação do profissional, coerente com as pesquisas de Oliveira (2008) e Oliveira et al. (2007).

No que se refere ao uso de estratégias de aprendizagem, os resultados demonstraram que a média dos alunos ingressantes foi um pouco maior que a dos concluintes, embora a diferença não seja significativa. Dados consonantes com a pesquisa de Figueiredo et al. (2016) que também não encontrou diferença significativa entre estudantes ingressantes e concluintes quanto ao uso de estratégias de estudo. Nos parágrafos seguintes serão explicitadas as correlações entre os construtos investigados, conforme apresentado na Tabela 3.

Tabela 3

Correlação entre as dimensões dos instrumentos para ingressantes e concluintes

$\begin{array}{ccc}\text { Compreensão } & \text { Atitudes } & \text { Estratégias de } \\ \text { de leitura } & \text { em leitura } & \text { aprendizagem }\end{array}$

\section{INGRESSANTES}

Compreensão de leitura

r

1

$\mathrm{p}$

Atitudes em leitura

$\mathrm{r}$

, 184

1

$\mathrm{p}$

, 131

Estratégias de aprendizagem $r$

, 035

, $485^{* *}$

1

$\mathrm{p}$

, 774

, 000

\section{CONCLUINTES}

Compreensão de leitura 


$\begin{array}{lcccc} & \mathrm{p} & & & \\ \text { Atitudes em leitura } & \mathrm{r} & , 145 & 1 & \\ & \mathrm{p} & , 386 & & \\ \text { Estratégias de aprendizagem } & \mathrm{r} & , 179 & , 567^{* *} & 1 \\ & \mathrm{p} & , 283 & , 000 & \\ * * \mathrm{p}<0,01 . & & & \end{array}$

No que concerne à relação entre as variáveis investigadas, não foi constatada correlação significativa entre o desempenho na compreensão leitora e nas escalas de atitude em leitura e de estratégias de aprendizagem. Esse resultado está em consonância com as pesquisas de Figueiredo et al. (2016) e Alcará e Santos (2013). Os autores atribuíram a falta de correlação ao baixo desempenho dos universitários no teste Cloze, ou seja, o baixo nível de compreensão leitora e o uso inadequado de estratégias de aprendizagem, já que apesar de os estudantes utilizarem as estratégias, seu uso pode ser insuficiente para compreensão de textos. Oliveira (2011) também não encontrou correlação significativa entre atitudes em leitura e o desempenho no Cloze. Ressalta-se a sugestão de Oliveira et al. (2007) de que sejam feitos novos estudos para se aferir validade concorrente entre a escala de atitudes de leitura e a habilidade de compreensão leitora. Em contraposição a esses achados, a pesquisa de Cantalice e Oliveira (2009) revelou uma correlação positiva e estatisticamente significativa entre estratégias de aprendizagem metacognitiva de leitura e o Teste Cloze, denotando que a compreensão textual é maior, quanto maior for o uso de estratégias de leitura. Dessa forma torna-se evidente a necessidade de novas pesquisas para investigar melhor a correlação entre esses construtos.

Em relação às estratégias de aprendizagem e às atitudes em leitura, foi constada uma correlação positiva, estatisticamente significativa nos dois grupos, indicando que quanto mais 
positiva é a atitude do estudante em relação à leitura maior é seu repertório de estratégias de aprendizagem. Dado importante, uma vez que ter atitudes positivas e utilizar melhores estratégias de estudo pode contribuir para uma melhor compreensão leitora e desempenho acadêmico. Destaca-se que não foram encontrados estudos que avaliassem a relação entre essas duas variáveis.

\section{Considerações finais}

Os resultados obtidos no presente estudo permitem a confirmação de algumas das hipóteses, como o baixo nível de compreensão leitora entre os universitários. O uso de estratégias de aprendizagem pode ser inadequado ou insuficiente para garantir a qualidade da aprendizagem. Não houve diferença entre os estudantes ingressantes e concluintes no nível de compreensão leitora, no uso de estratégias de aprendizagem e nas atitudes de leitura. Esses resultados confirmam a literatura revisada e estimulam uma reflexão sobre a qualidade da formação no ensino superior, pois o ideal seria que os alunos concluintes apresentassem melhor nível de compreensão de leitura devido aos anos de formação.

A ausência de correlação entre a compreensão leitora com as estratégias de aprendizagem e com as atitudes pode ser compreendida pelo baixo nível de compreensão em leitura. Entretanto, sugerem-se novas investigações acerca da relação entre essas e outras variáveis que possam predizer a compreensão leitora, bem como utilizar amostras compostas por pessoas com altos níveis de compreensão. Também poderiam ser desenvolvidas pesquisas com amostras maiores e mais representativas, envolvendo estudantes de diferentes cursos e regiões, além de incluir instituições de ensino privadas e públicas. Salienta-se que entre as limitações deste estudo estão as características da amostra, que se restringiu a um único curso de uma única instituição de ensino superior particular do interior de Minas Gerais, embora essa instituição receba alunos de diferentes municípios. 
Por fim, cabe destacar que o uso adequado de estratégias de aprendizagem, atitudes positivas diante da leitura e a capacidade de ler com autonomia, são fatores que, provavelmente, contribuem para a qualidade da formação dos futuros profissionais. Contudo, os estudantes estão ingressando no ensino superior com dificuldades básicas de compreensão de leitura, resultantes de uma formação anterior precária, e que representam obstáculos para formação profissional. Assim, torna-se cada vez mais urgente que as universidades considerem a necessidade da realização de diagnóstico das dificuldades de seus ingressantes para implementação de programas de intervenção, de forma curricular ou extracurricular, que possam auxiliar os estudantes a superarem as suas dificuldades para melhorar suas habilidades cognitivas, ampliando o repertorio de estratégias de aprendizagem e desenvolvendo a capacidade de leitura independente. Dessa forma, a comunidade acadêmica estará preparando os alunos para o constante desenvolvimento pessoal e atuação profissional.

Algumas estratégias podem ser implementadas pelas universidades para melhorar o nível de compreensão e as atitudes de leitura dos estudantes, como por exemplo, a realização de diagnóstico para identificar as dificuldades dos alunos no início do curso e a partir dos resultados podem ser criadas oficinas de nivelamento, círculos de leitura, cursos de extensão, leituras orais em sala de aula, entre outras (Alcará \& Santos, 2015; Calderon-Ibanez \& Quijano-Penuela, 2010). Este estudo foi mais uma contribuição com a intenção de estimular novas pesquisas acerca da compreensão leitora em universitários, bem como inspirar reflexões acerca da necessidade de desenvolver a competência de leitura na educação básica, e ressaltar a importância de intervenções de remediação no ensino superior, visando à qualidade da formação dos universitários. 


\section{Referências}

Alcará, A. (2012). Compreensão de leitura, estratégias de aprendizagem e motivação em universitários: estudos de validade de medidas. Tese de Doutorado, Universidade São Francisco, Itabira. Recuperado em 06 de setembro de 2018, de http://revistaseletronicas.pucrs.br/ojs/index.php/revistapsico/article/view/12258.

Alcará, A., \& Santos, A (2013). Compreensão de leitura, estratégias de aprendizagem e motivação em universitários. Psico, 44(3), 11-420. Recuperado em 04 de fevereiro de 2018, de http://revistaseletronicas.pucrs.br/ojs/index.php/revistapsico/article/view/12258/10416.

Alcará, A., \& Santos, A. (2015). Avaliação e desenvolvimento da compreensão de leitura em universitários. Estudos $\quad$ de Psicologia $\quad$ (Campinas), 32(1), $\quad$ 6373. https://dx.doi.org/10.1590/0103-166X2015000100006.

Bortoletto, D., \& Boruchovitch, E. (2013). Learning Strategies and Emotional Regulation of Pedagogy Students. Paidéia $\quad$ (Ribeirão $\quad$ Preto), 23(55), 242. https://dx.doi.org/10.1590/1982-43272355201311.

Calderón-Ibáñez, A., \& Quijano-Peñuela, J. (2010). Características de comprensión lectora en estudiantes universitarios. Estudios Socio-Jurídicos, 12(1), 337-364. Recuperado em 14 de setembro de 2019, de: http://www.scielo.org.co/scielo.php?script=sci_arttext\&pid=S012405792010000100015\&lng=en\&tlng=es.

Cantalice, L., \& Oliveira, K. (2009). Estratégias de leitura e compreensão textual em universitários. Psicologia Escolar e Educacional, 13(2), 227-234. Recuperado em 14 de setembro de 2019, de: http://pepsic.bvsalud.org/scielo.php?script=sci_arttext\&pid=S1413$85572009000200004 \& \operatorname{lng}=$ pt\&tlng=pt. 
Figueiredo, A.; Minervino, C.; Pereira, E., \& Silveira, N. (2016). Compreensão leitora e estratégias de estudo: estudo correlacional com universitários. Psicologia Argumento 34(86), 218-229. Recuperado em 11 de outubro de 2017, de http://www2.pucpr.br/reol/pb/index.php/pa?dd1=16428\&dd99=view\&dd98=pb.

Gonçalves, S. (2008). Aprender a ler e compreensão do texto: processos cognitivos e estratégias de ensino. Rev. Iberoamericana de Educación. (46), 135-151. Recuperado em 12 de outubro de 2017, de: http://rieoei.org/rie46a07.htm.

Lawi, E. (2015). Perfil de hábitos de leitura e qualidade de vida de alunos ingressantes em 2014, de odontologia e fonoaudiologia da Faculdade de Odontologia da USP de Bauru, São Paulo. Dissertação de Mestrado, Faculdade de Odontologia de Bauru, Universidade de São Paulo, Bauru. doi:10.11606/D.25.2015.tde-24112015-161003. Recuperado em 2019-09-14, de $\underline{\text { www.teses.usp.br }}$

Maher, J., \& Silva, E. (1983) Desenvolvimento de uma escala para determinar atitudes de leitura. Educação e Seleção. (8), 54-71. Recuperado em 14 de setembro de 2019, de http://publicacoes.fcc.org.br/ojs/index.php/edusel/article/view/2557/2510.

Mohallen, R. (2016). Estratégias de Aprendizagem em universitários: avaliação e intervenção. Tese de Doutorado em Psicologia, Universidade São Francisco - USF/SP, Itatiba. Recuperado em 28 de fevereiro de https://www.usf.edu.br/galeria/getImage/427/17279032934113009.pdf.

Oliveira, K. (2008). Compreensão da leitura, atitudes de leitura e desesperança em universitários. Psicologia: $\quad$ Ciência $\quad$ e $\quad$ Profissão, 28(4), $\quad$ 820831. https://dx.doi.org/10.1590/S1414-98932008000400013. 
Oliveira, K. (2011). Considerações acerca da compreensão em leitura no ensino superior. Psicologia: Ciência e Profissão, 31(4), 690-701. https://dx.doi.org/10.1590/S1414$\underline{98932011000400003 .}$.

Oliveira, K.., Cunha, N., \& Santos, A. (2015). Compreensão de leitura no curso de Psicologia: explorando diferenças. Psicologia Ensino \& Formação, 6(2), 87-100. Recuperado em 14 de setembro de 2019, de http://pepsic.bvsalud.org/scielo.php?script=sci_arttext\&pid=S2177$\underline{20612015000200007 \& \operatorname{lng}=p t \& t \operatorname{lng}=\mathrm{pt} .}$

Oliveira, R., \& Oliveira, K. (2007). Leitura e condições de estudo em universitários ingressantes. Psic: revista da Vetor Editora, 8(1), 51-59. Recuperado em 14 de setembro de 2019, de $\quad$ http://pepsic.bvsalud.org/scielo.php?script=sci arttext\&pid=S1676$\underline{73142007000100007 \& \operatorname{lng}=\text { pt\&tlng=pt. }}$

Oliveira, K., \& Santos, A. (2005). Compreensão em leitura e avaliação da aprendizagem em universitários. Psicologia: $\quad$ Reflexão $\quad e \quad$ Crítica, 18(1), 124. https://dx.doi.org/10.1590/S0102-79722005000100016.

Oliveira, K., \& Santos, A. (2008). Estudo de intervenção para a compreensão em leitura na universidade. Interação em Psicologia, 12(2). doi: http://dx.doi.org/10.5380/psi.v12i2.9575.

Oliveira, K., Santos, A., \& Oliveira, E. (2007). Estudo das propriedades psicométricas de uma escala de atitudes de leitura para universitários. Paradígma, 28(2), 165-180. Recuperado em 14 de setembro de 2019, de http://ve.scielo.org/scielo.php?script=sci_arttext\&pid=S1011$\underline{22512007000200008 \& \operatorname{lng}=\text { en\&tlng=pt. }}$

Rodrigues, A. (1973). Conceito de Atitude. In: Psicologia Social. 3 ed. Petrópolis: Vozes, cap. 12, p. 393-409. 
Santos, A., Vendramini, C., Suehiro, A., \& Santos, L. (2006). Leitura compreensiva e utilização de estratégias de aprendizagem em alunos de Psicologia. Estudos de Psicologia (Campinas), 23(1), 83-91. https://dx.doi.org/10.1590/S0103-166X2006000100010.

Santos, A. (1997). Psicopedagogia no $3^{\circ}$ grau: avaliação de um programa de remediação em leitura e estudo. Pro-Posições, 8(1), 27-37. Recuperado em 9 de outubro de 2017 de https://periodicos.sbu.unicamp.br/ojs/index.php/proposic/article/view/8644201.

Santos, A., Suehiro, A., \& Oliveira, K. (2004). Habilidades em compreensão da leitura: um estudo com alunos de psicologia. Estudos de Psicologia (Campinas), 21(2), 2941. https://dx.doi.org/10.1590/S0103-166X2004000200003.

Santos, A., Primi, R., Taxa, F., \& Vendramini, C. (2002). O teste de Cloze na avaliação da compreensão em leitura. Psicologia: Reflexão e Crítica, 15(3), 560. https://dx.doi.org/10.1590/S0102-79722002000300009.

Santos, A. (2004). O Cloze como técnica de diagnóstico e remediação da compreensão em leitura. Interação em Psicologia, 8(2). doi :http://dx.doi.org/10.5380/psi.v8i2.3257.

Silva, L. (2012). Estilos e estratégias de aprendizagem de estudantes universitários. Dissertação, Instituto de Psicologia, Universidade de São Paulo, São Paulo. doi: doi:10.11606/D.47.2012.tde-12092012-161445. Recuperado em 2019-09-14, de www.teses.usp.br.

Silva, E., \& Witter, G. (2008). Compreensão de texto e desempenho acadêmico em estudantes de psicologia. Estudos $\quad$ de $\quad$ Psicologia $\quad$ (Campinas), 25(3), $\quad 395-$ 403. https://dx.doi.org/10.1590/S0103-166X2008000300008. 
Souza, L. (2010). Estratégias de aprendizagem e fatores motivacionais relacionados. Educar em Revista, (36), 95-107. Recuperado em 27 de outubro de 2018, de http://www.redalyc.org/articulo.oa?id=155015820008.

Sternberg, R. J. (2000). Percepção. In: Psicologia Cognitiva. Porto Alegre: Atmed, cap. 4, p. 109148.

Tinajero, C., Lemos, S., Araújo, M., Ferraces, M., \& Páramo, M. (2012). Cognitive style and learning strategies as factors which affect academic achievement of brazilian university students. Psicologia: Reflexão e Crítica, 25(1), 105-113. https://dx.doi.org/10.1590/S0102$\underline{79722012000100013 .}$.

Recepción: 13 de noviembre de 2019

Última Revisión: 23 de diciembre 2019

Aceptación Final: 30 de diciembre 2019 